\title{
QuANTIFying Vertical FluXes fRom TuRbulence IN THE OCEAN
}

\author{
By J.N. Moum
}

$\mathrm{T}$ URBULENCE REPRESENTS the smallest scales of the oceanic flowfield, ranging from the order of $1 \mathrm{~mm}$ diffusive scales to eddy scales on the order of $1 \mathrm{~m}$ in the thermocline, to $10 \mathrm{~m}$ in the wind-mixed upper ocean, and to $100 \mathrm{~m}$ in the most energetic tidal mixing or convectively mixing flows. Although models of the general circulation cannot possibly resolve the scales of turbulence, its effects must be accounted for. This is because turbulence is the primary agent for irreversibly mixing mass, heat, nutrients and other scalar properties important to the ocean's stratification, to its lifeforms, and to its ability to rid itself of pollutants. By stretching material surfaces, turbulence acts to enhance concentration gradients so that molecular diffusion can proceed rapidly enough to play a role in the largescale, irreversible redistribution of properties.

Because the ocean is stably stratified, the largest property gradients are in the vertical direction. The biggest effect of turbulence is to enhance vertical fluxes of properties. It is critical that we quantify these vertical fluxes. This has been attempted from indirect inferences based on the large-scale flowfield, from measurements of the end effects of turbulence-enhanced diffusion and from direct observations of properties of the turbulence itself.

Vertical fluxes may be inferred from the larger scale circulation's need to make ends meet (Garrett, 1994). Largescale budget equations consistently require more turbulent mixing in the main thermocline and below than has been observed (Munk, 1966). So do large-scale

J.N. Moum. College of Oceanic and Atmospheric Sciences. Oregon State University. Corvallis. OR 97331, USA. numerical models, in which physical processes on scales smaller than can be resolved by the model-typically on the order of $100 \mathrm{~m}$ vertically and $10 \mathrm{~km}$ horizontally-are simply parameterized. The parameterized physics includes turbulence.

The only direct measure of the vertical fluxes due to turbulence comes from observations of the temporal changes in vertical distributions of scalar variables. For example, the change in the center of mass of a stratified fluid exposed only to turbulence-enhanced mixing represents the system's gain in potential energy. The fact that other processes move density surfaces vertically in the ocean (and yet do not result in irreversible energy transfer). makes this measurement difficult. What we specifically want to know is the scalar flux across density surfaces, that is the diapycnal flux (I use the term vertical flux here as a general approximation). A means to determine the scalar flux across density surfaces has been devised by Ledwell et al. (1993), who injected dye on a density surface in the thermocline and observed its spreading over periods of a year or so. Although a measure of the end result of the turbulence is obtained, this method does not directly measure the properties of the turbulence itself, nor does it tell us about the specific processes involved.

Indirect estimates of vertical fluxes come from direct measurements of some properties of the turbulence (or in oceanographer's parlance, microstructure). These include flux estimates based on measurement of the temperature variance dissipation rate, $\chi$ (Osborn and Cox, 1972), estimates based on the turbulent kinetic energy dissipation rate. $\epsilon$ (Osborn. 1980), and conservation equation balances. The dissipation estimates require resolution of temperature fluctua- tions to scales of a few millimeters or of velocity fluctuations to scales of a few centimeters. Another estimate, based on a mixing length analogy, uses information about the energy-containing eddies of the turbulence (Moum, 1996a), which in the main thermocline requires resolution of velocity fluctuations at $-10 \mathrm{~cm}$ scale. It should be emphasized that these microstructure flux estimates are not flux measurements. The assumptions leading to these flux estimates are fraught with uncertainty.

In the Reynolds-averaged equations. the vertical flux of a scalar $C$ is the average value of the correlation between the vertical velocity fluctuation and the scalar fluctuation, $\left\langle w^{\prime} C^{\prime}\right\rangle$. This is referred to as the eddy-correlation flux. It has been measured by the atmospheric boundary layer community since the $1950 \mathrm{~s}$, and is now measured using commercially available instrumentation. Such measurements produced classical results in the 1960s, for example, in the Kansas experiment (Haugen et al., 1971). Only in the 1990s have we seen equivalent types of eddycorrelation measurements in the ocean made by a few groups using homemade instrumentation. This lag in oceanic flux measurements is due to the difficulties of making such measurements in the ocean (including platform motion, internal wave contamination and small signal levels) and to the fact that the smaller scales of oceanic turbulence dictate finer measurement resolution.

\section{Complexity of Small-Scale Flowfields}

Although it will be beyond our abilities for the foreseeable future to image the flowfield on all of the scales of motion, in time and three spatial dimensions. we can gain an appreciation for the richness of the small-scale flowfield from numerical simulations, even though numeri- 
cal simulations are limited by finite computer memory in the range of scales they can resolve. Fully resolved simulations, termed direct numerical simulations (DNS), require resolution of the smallest scales of the flowfield. This puts an upper limit on the size of scales that can be resolved. Current computing capabilities permit full resolution of only $\sim 10-\mathrm{cm}$ thick patches of turbulence representative of the smallest turbulent events in the ocean thermocline (Moum, 1996a). An alternative is large eddy simulation (LES), designed to resolve the energycontaining eddies of the flow, but also to parameterize the smallest scales of the turbulence that actually accomplish the irreversible mixing.

A sample LES intended to represent the response of the upper ocean in the western equatorial Pacific to a westerly wind burst indicates the range of scales excited (E.D. Skyllingstad, W.D. Smyth, J.N. Moum and H. Wijesekera, unpublished). Shown is a two-dimensional slice at one point in time of a three-dimensional, temporally varying flowfield. The domain is $384 \mathrm{~m} \times 384$ $\mathrm{m}$ horizontally and $96 \mathrm{~m}$ vertically, with a uniform grid spacing of $1.5 \mathrm{~m}$ and time step of $1.2 \mathrm{~s}$.

The subsample of simulated data in Figure 1 shows vertical velocity, w, temperature, T, and $\epsilon$. The length scales evident in $w$ range from a few meters to the depth of the boundary layer. In the ocean, the best in situ observation we could make of this field would either trace a single vertical, or perhaps horizontal line. Such observations are blind to the flowfield $1 \mathrm{~m}$ to either side. For operational reasons, it is not possible to repeat vertical profiles to $100 \mathrm{~m}$ depth until at best five min, or 250 time steps later. Looking closely at the section of T, one can see what appears to be an overturning wave $20 \mathrm{~m}$ into the section at 70 $\mathrm{m}$ depth. This feature is suggestive of a Kelvin-Helmholtz billow, the form of instability associated with stratified shear flow. The billow appears at the base of the mixed layer, where the fluid is sufficiently stratified that the energy-containing scales of the turbulence are not well resolved by the LES. A fundamental limitation of LES is its inability to resolve the smaller energy-containing scales in stratified flows. This limitation has restricted the use of LES in strongly stratified flows, although it has been widely used in studies of convecting boundary
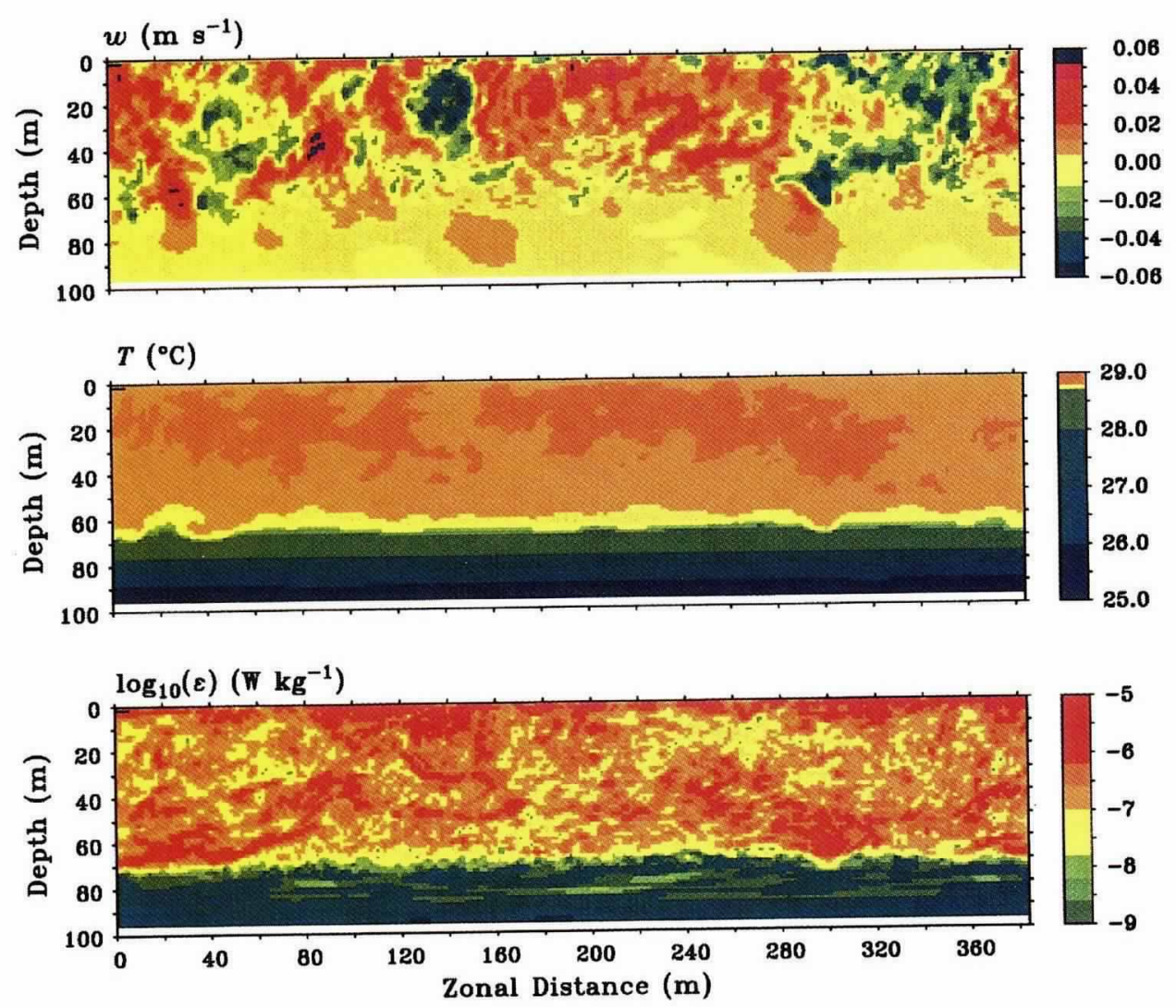

Fig. 1: Depth-zonal section of vertical velocity, $w$, temperature, $T$, and $\log \epsilon$, from a large eddy simulation of the upper ocean's response to a westerly wind burst in the western equatorial Pacific. Initial conditions and surface forcing were derived from observations taken during the Coupled Ocean Atmosphere Response Experiment (COARE), from E.D. Skyllingstad, W.D. Smyth, J.N. Moum and H. Wijesekera (unpublished).

layers (especially in the atmosphere) where the energy-containing scales are of the order of the boundary layer thickness.

Another view of the complexity of the small-scale flowfield comes from observations. During the Tropical Instability Wave Experiment in fall 1991, two ships maintained station within several kilometers of each other for a period of 3.5 days (Moum et al., 1995). Approximately 1,000 turbulence profiles were made and $\epsilon(\mathrm{z})$ computed for each. Time series of 5-m vertical averages centered at $50 \mathrm{~m}$ depth, well below the mixed layer at that time, indicate that the long-term trends, dominated by daily variations, are duplicated in records from each data set (Fig. 2). Mean profiles over the 3.5-day period agree within confidence limits, but daily averages do not. The lack of agreement on daily time scales results from variations of several orders of magnitude in $\epsilon$ and several hours in duration that can be seen in Figure 2. This indicates very different events simultaneously taking place at the two closely spaced locations. Note that the spacing between the two ships is not that much greater than the horizontal extent of the simulation shown in Figure 1.

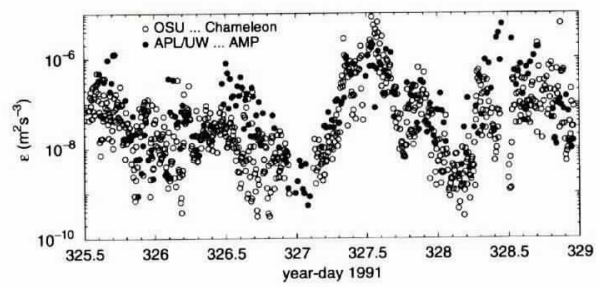

Fig. 2: Time series of $\epsilon$ at $50 \mathrm{~m}$ depth made by two different groups from vertical profilers on two separate ships located within a few $\mathrm{km}$ of each other. OSU, Oregon State University using their profiler Chameleon; APLUW, Applied Physics Lab/University of Washington using their profiler AMP. From Moum et al. (1995). 


\section{What Do Large-Scale Flow Modelers Want from Us?}

As indicated by the two examples discussed above, insufficient resolution of the space and time variability of the small-scale flowfield will continue to haunt our interpretations of measurements for the foreseeable future. How can we make a reasonable contribution to the problem confronted by large-scale flow modelers? Modelers require an estimate of fluxes of properties across their grid cells due to processes occuring at scales smaller than their grid size. These fluxes are usually parameterized by the product of a turbulent diffusion coefficient and a property gradient. In many instances, large-scale modelers can do nothing about the physics at scales smaller than their model's grid size other than to assign a value to a turbulent diffusion coefficient. This value may be varied from grid cell to grid cell and in time, according to some specified parameterization. But it remains an oversimplification of the real physics that will include, as well as the three-dimensional turbulence. Langmuir circulations near the surface, internal gravity waves propagating throughout the volume, surface waves breaking at the free surface, and shear instability in the interior of the fluid, to name a few of the physical processes we recognize.

It seems likely that current choices of subgrid scale parameterization range from bad to worse. A reasonable goal for the investigators of small-scale ocean physics is to gain an understanding that is at least sufficient to avoid the worst choices.

\section{Examples of Flux Measurements}

Examples of flux measurements using very different methods give us quite different insights.

\section{Tracer Releases}

A direct measure of the vertical (really diapycnal) flux is obtained by observing the spreading of a purposely introduced artificial tracer. This has been done in several locations by Jim Ledwell and coworkers and has provided a very important result in the North Atlantic Tracer Release Experiment (NATRE; Ledwell $e t$ al., 1993). The rate of vertical spreading can be interpreted in terms of an eddy diffusion coefficient, $K_{v}$. The estimate of $\mathrm{K}_{v}$ from NATRE of $\sim 10^{-5} \mathrm{~m}^{2} \mathrm{~s}^{-1}$ at $300 \mathrm{~m}$ depth appears to confirm estimates of $K_{v}$ from microstructure data obtained in the thermocline over the past 20 years. It also agrees with microstructure estimates of $\mathrm{K}_{\mathrm{v}}$ made during the NATRE experiment itself, but is about 10 times smaller than that inferred from large-scale budgets and numerical models. Such a small value of $\mathbf{K}_{v}$ strongly suggests that most mixing in the upper part of the thermocline does not happen in situ but occurs while fluid is in contact with the surface, after which it is stirred along sloping density surfaces (Garrett, 1993).

What we learn from tracer release experiments is the average rate of mixing between two endpoints in time over a region many times larger than individual mixing events. What we do not learn is how this mixing was achieved, nor even how it evolved between the two endpoints. Consequently, we can only guess at the processes responsible for the mixing. In light of atmospheric and oceanic variability on decadal and longer time scales, we require a better understanding of the physics responsible for the mixing so that we can have better predictive capabilities. A first attempt at this, at least in the thermocline, is the internal wave scaling first proposed by Gregg (1989) and since revised by others.

\section{Eddy-Correlation Measurements}

In the past few years, several groups have found ways to measure eddy-correlation fluxes from horizontal tows (Yamazaki and Osborn, 1993; Fleury and Lueck, 1994; Gargett and Moum, 1995). These measurements are akin to those obtained by flying through the atmospheric boundary layer with a rack of probes mounted on the nose of an aircraft. Figure 3 shows an example of such a record derived from a vehicle towed behind a ship at speeds of $0.7-1.5 \mathrm{~m} \mathrm{~s}^{-1}$ at $\sim 400 \mathrm{~m}$ depth east of Barbados (Fleury and Lueck, 1994). Temperature fluctuations are on the order of $0.01^{\circ} \mathrm{K}$, and vertical velocity fluctuations are several $\mathrm{mm} \mathrm{s}^{-1}$. Their product is the instantaneous eddycorrelation heat flux, $w^{\prime} \theta^{\prime}$. Along this tow, instantaneous values of $w^{\prime} \theta^{\prime}$ may have either sign, positive values representing restratification of displaced fluid parcels, and negative values representing down-gradient transport, that is, either downward transport of warm fluid or upward transport of cool fluid. Down-gradient transport increases system potential energy by moving mass upward. This must be the end result of mixing. A reli- able estimate of net flux requires averaging many down- and counter-gradient instantaneous values.

In a stratified fluid, we usually find turbulent patches much greater in horizontal extent than vertical. This means that a longer record is obtained from a horizontal pass through a patch than from a vertical pass. We therefore expect to obtain more degrees of freedom and smaller confidence limits in the estimates made from data obtained in each patch from horizontal tows. However, in the thermocline. where turbulence occurs intermittently, fewer patches per unit length of record will be sampled by horizontal tows. More important, it is the vertical divergence of the flux that we really want to know, so there is good reason to try to make sense of eddy-correlation measurements obtained from vertical protiles.

An example of a vertical profile of eddy-correlation flux measurement comes from about the same depth as the horizontal tow shown in Figure 3, but 1,000 nautical miles off northern California (Fig. 4, from Moum, 1996b). Temperature and vertical velocity fluctuations are about the same magnitude as those shown in Figure 3. The length of record through the patch is $\sim 10$ times smaller, however, so confidence limits are considerably larger.

Typical signal levels obtained from aircraft flying through the atmospheric boundary layer over the ocean are on the order of $1^{\circ} \mathrm{K}$ temperature fluctuation and $1 \mathrm{~m} \mathrm{~s}^{-1}$ vertical velocity fluctuation (Friehe, 1986). The comparatively smaller signals in the ocean thermocline highlight one of the difficulties in making these measurements.

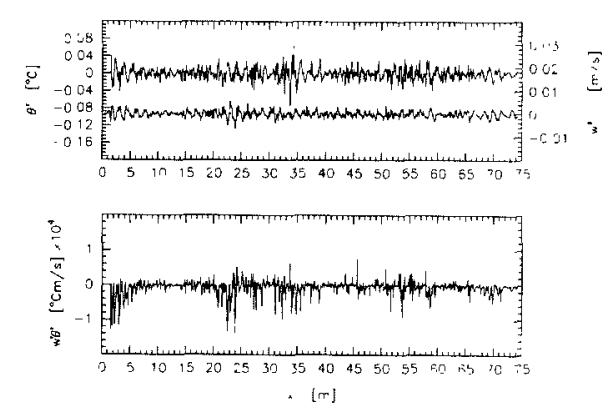

Fig. 3: Nearly horizontal space series of fluctuations of temperature, $\theta^{\prime}$, vertical velocity, $w^{\prime}$, and their product $w^{\prime} H^{\prime}$. The series of $\theta^{\prime}$ and $w^{\prime}$ have been highpass filtered at $0.5 \mathrm{cpm}$. From Fleury and Lueck (1994). 


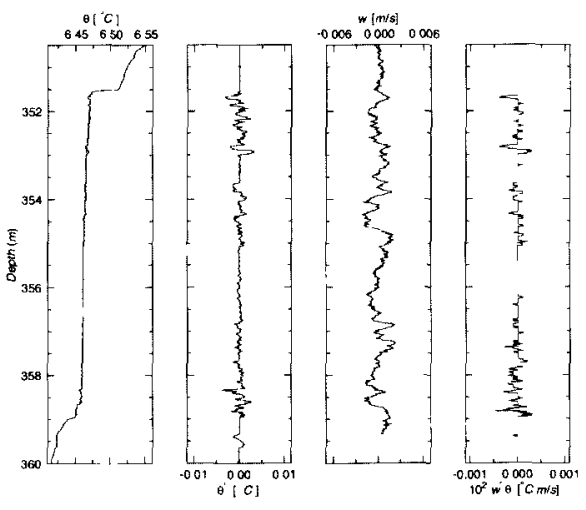

Fig. 4: Vertical profiles of $\theta$ (instantaneous, solid; reordered, dashed), temperature fluctuation, $\theta^{\prime}$ (the difference between the two $\theta$ curves), $w^{\prime}$, and $w^{\prime} \theta^{\prime}$. From Moum (1996b).

\section{Prospects for Understanding Small-Scale Flowfields}

One way to summarize what types of small-scale flowfields have been sampled in the ocean and what range of scales is involved in the turbulence is a plot of $\log$ $\epsilon$ versus $\log N$ (Fig. 5). The scales of the turbulence in a stratified fluid range from the energy-containing scale, set by buoyancy forces in stratified turbulence as $\mathrm{L}_{0}$ $=\left(\epsilon / N^{3}\right)^{1 / 2}$ (dashed lines rising to the right in Fig. 5) to the diffusive scale $\left(\mathrm{vD}^{2} / \epsilon\right)^{1 / 4}$ (horizontal dashed lines in Fig. 5). Here. $\mathrm{N}$ is the buoyancy frequency, $\mathrm{v}$ is the kinematic viscosity and $D$ is replaced by either $v$ for velocity, or by the molecular diffusivity for temperature, $\mathrm{D}_{\mathrm{T}}$, or salinity, $D_{S}$. This means that scalars such as temperature and salinity vary on length scales smaller than the smallest length scales of velocity variations by a factor $\mathrm{L}_{\text {ielocil }} / \mathrm{L}_{\text {cualal }}=\sqrt{\mathrm{v} / \mathrm{D}}$. In seawater, the Prandtl number, $\operatorname{Pr}=\mathrm{v} / \mathrm{D}_{\mathrm{T}}=7$ and Schmidt number $\mathrm{Sc}=\mathrm{D}_{\mathrm{S}} / \mathrm{D}_{\mathrm{T}}=100$. The smallest scales in temperature are $\sqrt{\mathrm{Pr}}$ times smaller than those in velocity, whereas the smallest scales in salinity are another $\sqrt{\mathrm{Sc}}$ times smaller still. The examples shown in Figure 5 are intended to be representative rather than inclusive. In some of the observational examples, the energy-containing eddies may be affected by proximity to boundaries as well as by stratification.

We can see the range of turbulence flowfields that have been observed, from the very energetic special cases at the top of Figure 5 to the more general situations typical of the upper ocean, thermocline and abyssal ocean. Unstratified, convectively mixed layers are off to the left. The range of scales excited by turbulence in tidal channels ranges from the order of $100 \mathrm{~m}$ to diffusive scales of sub-millimeter size-a range of over 5 orders of magnitude. The large range in scales makes such a flowfield impossible to represent with current computing power.

However, in weaker, more stratified flows, such as the weakest cases typical of the deep ocean, where $\mathrm{L}_{\mathrm{o}} \sim 0.1 \mathrm{~m}$ and diffusive scales for $\mathrm{T}$ are approximately $0.001 \mathrm{~m}$, it becomes feasible to represent turbulent patches with $\operatorname{Pr}=7$. Although typically turbulence simulations are run with $\operatorname{Pr}=1$, which is correct for air, there is reason to believe that physical misinterpretations can result if the correct $\mathrm{Pr}$ is not used for seawater (W. Smyth, personal communication). At present, it is not possible to consider the small salinity scales, because $\mathrm{Sc}$ is so large.

The ratio of energy-containing length scale to diffusive length scale is $\left(\epsilon / \mathrm{NN}^{2}\right)^{1 / 41}$. The cube of this quantity is di-

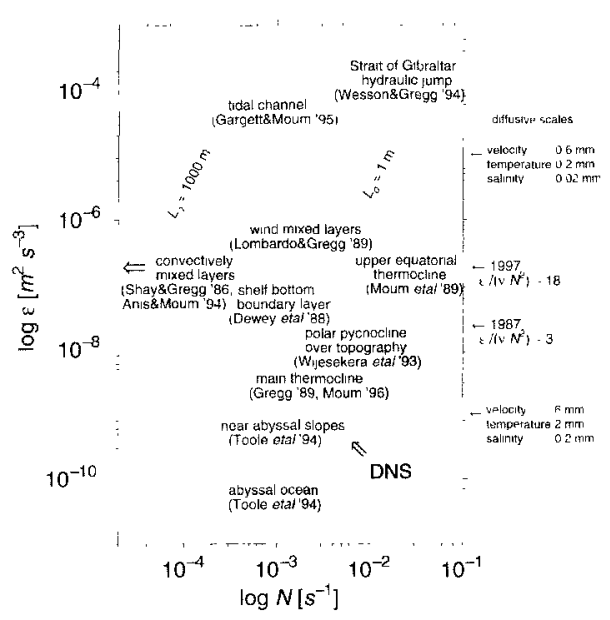

Fig. 5: Log-log plot of turbulent kinetic energy dissipation rate, $\epsilon$, versus buoyancy frequency, $N$. The range covers most of the observed cases from the ocean, representative examples of which are noted. The energy-containing scale, $L_{0}=\left(\epsilon / N^{*}\right)^{1 / 2}$, is represented by dashed lines sloping up to the right and is plotted at decade intervals. The small, diffusile scales. $\left(v D^{2} / \epsilon\right)^{1 / 4}$, are represented by horisontal dashed lines. The diffusive scales for velocity, temperature, and salinity for $\epsilon=10^{-4} \mathrm{~m}^{2} \mathrm{~s}^{-4}$ and $\epsilon=10^{4} \mathrm{~m}^{2}$ $s^{-3}$ are noted at right. At any point on this curve, one can infer the range of scales in a stratified turbulent flow. The shaded region represents current capabilities for computing fully resolved, $\mathrm{Pr}$ $=7$ turbulence. rectly proportional to the computational memory requirement for simulations. Present and 1987 capabilities are noted at the bottom right of Figure 5, and the shaded region denotes present capabilities in $\epsilon-\mathrm{N}$ space. Although flowfields cannot be represented, and only a single turbulent patch can be simulated, it is now possible to observe the complete life cycle, growth through decay, of turbulence representative of the weakest cases observed in the abyss and thermocline. One way these simulations are being used is to test the representativeness of our limited ability to sample the smallest scales within the context of a fully resolved three-dimensional temporally varying turbulence.

At the present rate of increase of computing power, extrapolated from the two data points in Figure 5, it is clear that we should not expect to see representative simulations of all the scales of turbulent patches even in the thermocline, within our careers. Probably the way we are going to learn more about the small-scale flowfields is to obtain better and more comprehensive measurements, especially those including the role of the initial instability in creating the turbulence.

\section{Acknowledgements}

Doug Caldwell. Ann Gargett, Rolf Lueck. Jonathan Nash and Bill Smyth all contributed thoughtful comments on early drafts of this paper. Thanks to Rolf Lueck for providing a copy of Figure 3 and to Ann Gargett for a well-organized 1997 TOS session in Seattle.

\section{References}

Anis. A. and J.N. Moum. 1994: Prescriptions for heat flux and entrainment rate in the upper ocean during convection. J. Phys. Oceanogr., 24, 2142-2155.

Dewey, R.K.. P.H. Leblond and W.R. Crawford, 1988: The turbulent bottom boundary layer and its influence on local dynamics over the continental shelf. Dyn. Atm. Oceans, 12. $143-172$.

Fleury, M. and R.G. Lueck, 1994: Direct heat flux estimates using a towed vehicle. J. Phys. Oceanogr., 24, 801-818.

Friehe. C.A.. 1986: Fine-scale measurements of velocity, temperature and humidity in the atmospheric boundary layer. In: Probing the Atmospheric Boundary Layer, D.H. Lenschow. ed. Amer. Met. Soc., Boston, 29-38.

Gargett. A.E. and J.N. Moum, 1995: Mixing efficiencies in turbulent tidal fronts: results from direct and indirect measurements of density flux. J. Phys. Oceanogr., 25, 25832608.

Garrett, C.J.R., 1993: A stirring tale of mixing. $\mathrm{Na-}$ ture. 364, 670-671. 
Garrett. C.J.R., 1994: Dispersion and mixing in the ocean. In: Ocean Processes in Climate Dynamics: Global and Mediterranean Examples, P. Malanotte-Rizzoli and A.R. Robinson, eds. Kluwer, Amsterdam, 61-77.

Gregg. M.C.. 1989: Scaling turbulent dissipation in the thermocline. J. Geophys. Res., 94, 9686 9698.

Haugen, D.A., J.C. Kaimal and E.F. Bradley, 1971: An experimental study of Reynolds stress and heat flux in the atmospheric boundary liayer. Q. J. Roy. Met. Soc., 97. 168-180.

Ledwell. J.R.. A.J. Watson and C.S. Law. 1993: Evidence for slow mixing across the pycnocline from an open ocean tracer release experiment. Nature, 364, 701-703.

Lombardo, C.P. and M.C. Gregg, 1989: Similarity scaling of viscous and thermal dissipation in a convecting surface boundary layer. $J$. Geophys. Res. 94, 6273-6284.
Moum. J.N., 1996a: Energy-containing scales of turbulence in the ocean thermocline. J. Gerphys. Res., 101, 14.095-14,109.

Moum. J.N., 1996b: Efficiency of mixing in the main thermocline. I. Geophys. Res., 101, 12,057-12.069.

Moum, J.N., D.R. Caldwell and C.A. Paulson. 1989: Mixing in the equatorial surface layer and thermocline. J. Geophys. Res., 94, 2005-2021

Moum. J.N., M.C. Gregg. R.C. Lien and M.E. Carr, 1995: Comparison of turbulence kinetic energy dissipation rate estimates from two ocean microstructure profilers. J. Atmos. Oceanic Technol., 12, 345-366.

Munk. W.H.. 1966: Abyssal recipes. Deep-Sea Res., 13, 707-730.

Osborn. T.R., 1980: Estimates of the local rate of vertical diffusion from dissipation measurements. J. Phws. Oceanogr., 10, 83-89.
Osborn, T.R. and C.S. Cox, 1972: Oceanic tine structure. Gcophss. Fluid Din., 3, 321345 .

Shay, T.J. and M.C. Gregg, 1986: Convectivelydriven turbulent mixing in the upper ocean. J. Phys, Oceanogr. 16, 1777-1798.

Toole, J.M., K.L. Polzin and R.W. Schmitt. 1994: Estimates of diapycnal mixing in the abyssial ocean. Science, 264, 1120-1123.

Wesson, J.C. and M.C. Gregg, 1994: Mixing in Camarinal Sill in the Strait of Gibraltar. $J$. Geophys. Res.. 99, 9847-9878.

Wijesekera. H.. L. Padman. T. Dillon. M. Levine. C. Paulson and R. Pinkel. 1993: The application of internal wave dissipation models to a region of strong mixing. J. Phys. Oc'e(mogr. 23. 269-286.

Yamazaki. H. and T.R. Osborn. 1993: Direct estimation of heat flux in the seasonal thermocline. J. Phys. Oceanogr. 23, 503-516. 